\title{
Significantly different clinical features between hypertriglyceridemia and biliary acute pancreatitis: a retrospective study of 730 patients from a tertiary center
}

Xiaoyao Li ${ }^{\dagger}$, Lu Ke ${ }^{\dagger}$, Jie Dong, Bo Ye, Lei Meng, Wenjian Mao, Qi Yang ${ }^{*}$, Weiqin Li ${ }^{*}$ (D) and Jieshou Li

\begin{abstract}
Background: Unlike western world, gallstones and hypertriglyceridemia (HTG) are among the first two etiologies of acute pancreatitis (AP) in China. But yet, detailed differences in clinical features and outcomes between hypertriglyceridemia and biliary acute pancreatitis have not been well described.

Methods: This retrospective study enrolled 730 acute pancreatitis patients from July 1, 2013 to October 1, 2016 in Jinling Hospital. The causes of the study patients were defined according to specific diagnostic criteria. The clinical features and outcomes of patients with hypertriglyceridemia acute pancreatitis (HTG-AP) and biliary acute pancreatitis (BAP) were compared in terms of general information, disease severity, laboratory data, system complications, local complications, and clinical outcome.

Results: In the enrolled 730 AP patients, 305 (41.8\%) were HTG-AP, and 425 (58.2\%) were BAP. Compared to BAP, the HTG-AP patients were found to be younger, with higher body mass Index (BMI), and much higher proportion of diabetes, fatty liver and high fat diet. Besides that, HTG-AP patients had significantly higher C-reactive protein (CRP) $(p<0.01)$ and creatinine $(p=0.031)$, together with more acute respiratory distress syndrome (ARDS) $(p=0.039)$, acute kidney injury (AKI) $(p<0.001)$, deep venous thrombosis $(p=0.008)$ and multiple organ dysfunction syndrome (MODS) ( $p=0.032$ ) in systematic complications. As for local complications, HTG-AP patients had significantly less infected pancreatitis necrosis $(p=0.005)$. However, there was no difference in mortality, hospital duration and costs between the groups.

Conclusion: HTG-AP patients were younger, more male, having high fat diet and with higher BMI compared to BAP patients. The prevalence of AKI/ARDS/DVT/MODS in HTG-AP patients was higher than BAP patients, while BAP patients had a greater possibility in development of infected pancreatitis necrosis (IPN). According to the multivariate analysis, only the complication of AKI was independently related with the etiology of HTG, however, BMI contributes to AKI, ARDS and DVT.
\end{abstract}

Keywords: Biliary acute pancreatitis, Hypertriglyceridemia acute pancreatitis, Acute kidney injury(AKI), Body Mass Index(BMI), Infected pancreatic necrosis

\footnotetext{
*Correspondence: yangqi_nj@163.com; njzy_pancrea@163.com

${ }^{\dagger}$ Xiaoyao Li and Lu Ke contributed equally to this work.

Surgical Intensive Care Unit (SICU), Department of General Surgery, Jinling

Hospital, Medical School of Nanjing University, Nanjing, China
}

(c) The Author(s). 2018 Open Access This article is distributed under the terms of the Creative Commons Attribution 4.0 International License (http://creativecommons.org/licenses/by/4.0/), which permits unrestricted use, distribution, and reproduction in any medium, provided you give appropriate credit to the original author(s) and the source, provide a link to the Creative Commons license, and indicate if changes were made. The Creative Commons Public Domain Dedication waiver (http://creativecommons.org/publicdomain/zero/1.0/) applies to the data made available in this article, unless otherwise stated. 


\section{Background}

Acute pancreatitis (AP) is an acute inflammatory disease which is characterized by local pancreatic inflammation and consequently systemic inflammatory response. The imaging of AP manifests as pancreatic edema or necrosis involving the pancreas as well as peripancreatic tissues and even distant organs $[1,2]$. In the western countries, the most common etiology of AP was gallstones, followed by alcohol abuse and hypertriglyceridemia [3, 4]. While the incidence of hypertriglyceridemia acute pancreatitis (HTG-AP) was much higher in China and has been increasing year by year according to the recent studies [5-10].

Previous studies well reported the clinical features of AP with different etiologies, rather than the detailed differences between HTG-AP and BAP. In this retrospective study from July 2013 to October 2016, we compared the clinical features, complications and outcomes between hypertriglyceridemia and biliary acute pancreatitis patients, as the two leading etiologies of acute pancreatitis in China.

\section{Methods}

\section{Patient selection}

This study retrospectively screened 999 AP cases admitted to the Surgical Intensive Care Unit (SICU), Department of General Surgery from July,1 2013 to October,1 2016, Jinling Hospital, Medical School of Nanjing University.

Patients who met the following criteria were excluded: (1) re-admission to the SICU; (2) traumatic, neoplastic, parathyroidal or other idiopathic pancreatitis; (3) younger than 18 years old. Eventually, 730 AP patients were enrolled in this study. The diagnosis and classification of the severity of AP were defined according to the 2012 revision of the Atlanta Classification.

\section{Data collection}

The data analyzed in this study included general information as sex, age, body mass index (BMI), diabetes, fatty liver, high fat diet, transfer from other hospitals and clinical features as pancreatitis severity, incidence of systemic and local complications, mortality. Levels of hemoglobin, hematocrit, platelet, C-reactive protein (CRP), IL-6, creatinine, alanine aminotransferase (ALT) and other laboratory results were included in the comparison. All the laboratory results were obtained from the Central Laboratory of Jinling Hospital according to the standard protocols. Meanwhile, acute physiology and chronic health evaluation II (APACHE II) score was manually calculated for each single patient.

According to the 2012 revised of the Atlanta Classification the etiology of AP and the definition of complications, including portal vein thrombosis, intra-abdominal hypertension and hemorrhage, deep vein thrombosis (DVT) and gastrointestinal fistula, were judged by two independent physicians [1]. Different severity of AP as mild, moderate and severe, was assessed based on the presence of local or systemic complications and transient/persistent organ failure [2].

\section{Definition}

The diagnosis of AP requires at least two of the following three features: (1) abdominal pain consistent with the disease (2) serum lipase activity (or amylase activity) at least three times greater than the upper limit of normal; and (3) characteristic findings from abdominal imaging [1].

The etiology of AP was analyzed by the following criteria. Biliary acute pancreatitis required the confirmation of gallstones or biliary sludge by any kind radiological imaging, including endoscopic ultrasonography(EUS), computed tomography (CT) and magnetic resonance cholangiopancreatography (MRCP), or elevated serum levels of ALT ( $>60 \mathrm{U} / \mathrm{L}$ ) and a BMI $<30 \mathrm{~kg} / \mathrm{m}^{2}$ indicates an episode of acute pancreatitis with a biliary origin [3]. Hypertriglyceridemia acute pancreatitis was confirmed by triglyceride levels $>1000 \mathrm{mg} / \mathrm{dL}$ or triglyceride levels between $500 \mathrm{mg} / \mathrm{dL}$ to $1000 \mathrm{mg} / \mathrm{dL}$ together with emulsion plasma and without any other obvious causes $[1,4]$.

The diagnosis of local complications was performed according to the 2012 revision of the Atlanta Classification. Infected pancreatitis necrosis (IPN) could be diagnosed by the presence of extra luminal gas in the pancreatic and/or peripancreatic tissues on CECT or by a positive bacterial culture of the necrosis from the fine-needle aspiration or drainage [1]. As this study being retrospectively, one clinical feature as "high fat diet" could not be defined exactly according to the standard definition. So here, we inquired the patients or their relatives to describe the normal typical 1-day diet. By calculating the constituent ratio, we defined the "high fat diet" as fat accounts over 30-35\%.

This study was performed according to the principles of the Declaration of Helsinki (modified 2000) and was approved by the institutional review board of Jinling Hospital.

\section{Statistical analysis}

We used SPSS 18.0 statistical software package (IBM Analytics, Armonk, NY) for statistical analyses. As the following tables show, datas were presented as median plus interquartile range (IQR) for continuous variables and absolute numbers and percentages for categorical variables. The $\mathrm{x}^{2}$ test was used for analyzing categorical variables and Student-test or Mann-Whitney test was used for analyzing continuous variables. Statistical significance was considered as a $P$ value of $<0.05$ (2-tailed). 


\section{Results}

\section{Baseline characteristics and clinical features}

Nine hundred and ninety-nine patients were initially screened and eventually 730 patients were enrolled. The guidelines and procedures were shown in Fig. 1. Firstly, 141 patients were excluded, who were re-admitted to ICU not because of AP but other complications, such as intestinal fistula, from the recovery ward. Secondly, the patients were divided into different groups by the etiology according to the revised Atlanta criteria, which were done by two independent physicians [1]. Traumatic acute pancreatitis patients $(n=9)$, parathyroidal acute pancreatitis patients $(n=1)$, idiopathic acute pancreatitis patients $(n=76)$, alcoholic acute pancreatitis patients $(n=37)$ were also exclude. After that, we got 426 biliary acute pancreatitis (BAP) patients and 309 hypertriglyceridemia acute pancreatitis (HTG-AP) patients. Thirdly, we excluded five patients with age $<18$, as 1 BAP patient and 4 HTG-AP patients. Ultimately, two groups were enrolled in this study, namely, biliary acute pancreatitis (BAP) $(n=425)$ and hypertriglyceridemia acute pancreatitis (HTG-AP) $(n=305)$ group.

The baseline characteristics of BAP and HTG-AP were displayed in Table 1. Compared to BAP patients, the HTG-AP patients were younger (40 vs $51, p<0.01$ ), with higher BMI (27 vs 22.7, $p<0.01$ ), and more males (214/ 91 vs $242 / 183, p<0.01)$, and higher incidence of diabetes $(32.1 \%$ vs $12.9 \%, p<0.01)$ and fatty liver $(43.9 \%$ vs $15.1 \%, p<0.01)$ and higher fat diet rate $(42.6 \% \mathrm{vs} 14.6 \%$, $p<0.01)$. Meanwhile, HTG-AP patients had significantly higher APACHEII score $(p<0.01)$ than BAP. Besides that, some similarities were also found between two groups, such as hypertension history, and duration from AP onset to transfer to our center.

All the patients initially received standard medical treatment according to the recent international guidelines [5]. Following a standard protocol (Additional file 1: Figure S1), fluid resuscitation was performed for each patient. The protocol includes the timing to initiation of hydration, rate of hydration and the appropriate solution. Moreover, $91 \mathrm{HTG}$-AP patients received apheresis.

According to the modified Atlanta Criteria, the AP patients were classified into mild acute pancreatitis (MAP), mild severe acute pancreatitis (MSAP), and severe acute pancreatitis (SAP). The percentages of MAP, MSAP, SAP were respectively $24,19.856 .2 \%$ in BAP, and 17.7, 28.9 and $53.4 \%$ in HTG-AP (Fig. 2).

\section{Systemic complications and laboratory data}

The laboratory data were displayed in Table 2, and systemic complications in Table 3 . The results suggested that more ARDS $(p=0.039)$, AKI $(p<0.001)$ and $\operatorname{MODS}(p=0.032)$ occurred in HTG-AP, together with significantly higher CRP $(p<0.01)$ and creatinine $(p=$ $0.031)$. Also, there were more deep venous thrombosis (DVT) $(27$ (6.4\%) vs 37 (12.1\%), $p=0.008)$ in HTG-AP than BAP. However, the other data were similar in two groups, as coagulation indexes of each group, hemoglobin, blood platelet, prothrombin time, activated partial thromboplastin time, and D-Dimer. $6(1.4 \%)$ BAP patients, but no HTG-AP patient, had suffered chylous fistula.

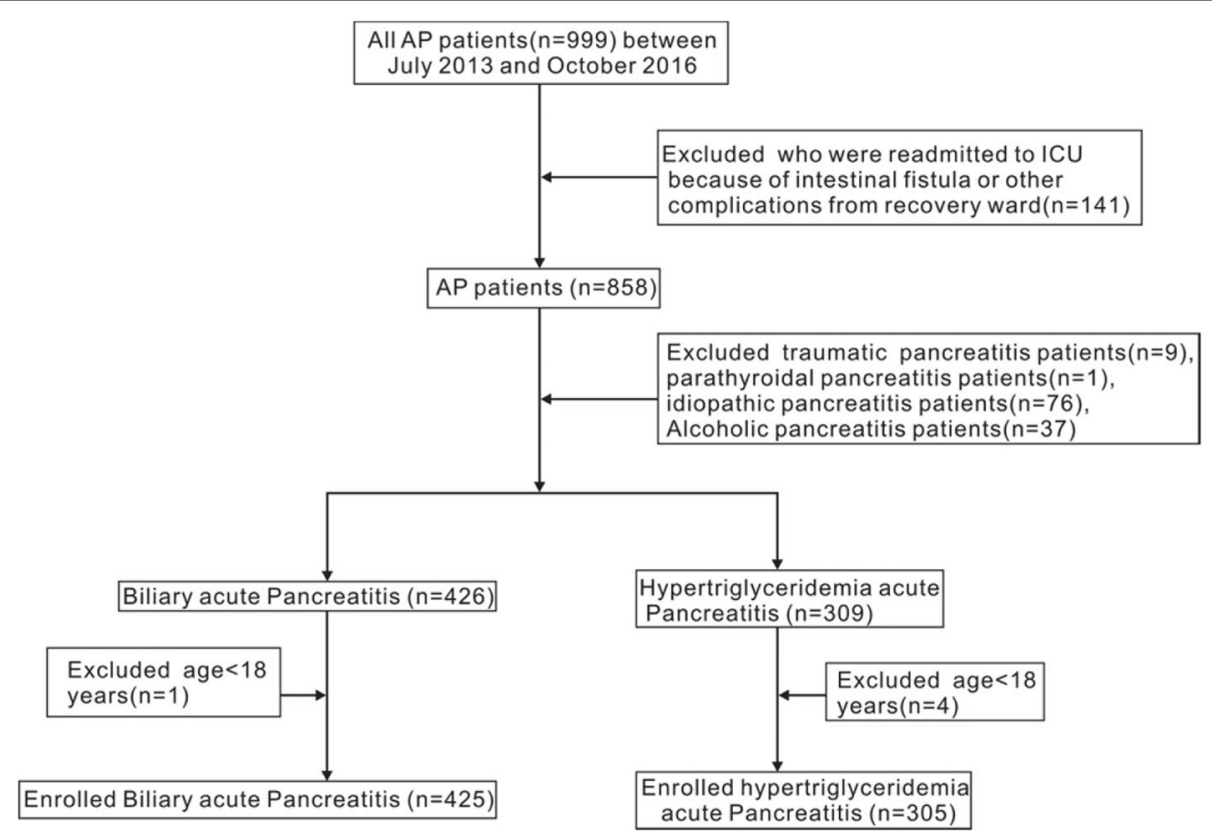

Fig. 1 Study selection for patients 
Table 1 Demographic data and baseline characteristics of the patients

\begin{tabular}{llll}
\hline Characteristic & $\begin{array}{l}\text { Biliary acute Pancreatitis } \\
(n=425)\end{array}$ & $\begin{array}{l}\text { Hypertriglyceridemia acute } \\
\text { Pancreatitis }(n=305)\end{array}$ & $P$ value \\
\hline Age, year & $51(43,64)$ & $40(33,47)$ & $214 / 91$ \\
Gender, male/female & $242 / 183$ & $27(24.9,30.4)$ & $P<0.01$ \\
BMI & $22.7(20.1,25.2)$ & $11(7,18)$ & $P<0.01$ \\
APACHE II score & $8(6,12.5)$ & $81(26.6 \%)$ & $P<0.01$ \\
Hypertension & $120(28.2 \%)$ & $98(32.1 \%)$ & 0.675 \\
Diabetes mellitus & $55(12.9 \%)$ & $134(43.9 \%)$ & $P<0.01$ \\
Fatty liver & $64(15.1 \%)$ & $130(42.6 \%)$ & $P<0.01$ \\
High fat diet & $62(14.6 \%)$ & $295(96.7 \%)$ & $P<0.01$ \\
Transfer from other hospitals & $406(95.5 \%)$ & $6(3,17)$ & 0.449 \\
Time taken for the patients transfer to our center after & $10(4,30)$ & & 0.541 \\
Onset of symptoms, Days & &
\end{tabular}

BMI body mass index, APACHE II Acute Physiology and Chronic Health Evaluation II

Then, we wonder if the significantly higher incidence of ARDS, AKI and DVT in HTG-AP was affected by only the etiology or other factors, such as age, gender, body mass index, diabetic mellitus, fatty liver and high-fat diet. So, we did the multivariate analysis to determine the association of ARDS/AKI/DVT with the etiology (Table 4). On multivariate logistic regression of ARDS/AKI/DVT adjusting for etiology, age, gender, body mass index, diabetic mellitus, fatty liver and high-fat diet, HTG-AP was found to be independently associated with more AKI, and higher BMI with more AKI, ARDS and DVT.

Then, we divided HTG-AP patients into three groups according to their TG level (peak TG level within $72 \mathrm{~h}$ of hospital admission with AP): group A: $\leq 10.2 \mathrm{mg} / \mathrm{dl}$ (less than the first quartile), group B: $10.3-21.9 \mathrm{mg} / \mathrm{dl}$ (between the first and third quartiles), and group $\mathrm{C}: \geq 22 \mathrm{mg} / \mathrm{dl}$ (more than the third quartile). The Cochran-Armitage trend test have been done to compare characteristics among three groups, results are displayed in Additional file 2: Table $\mathrm{S} 1$ and Additional file 3: Figure S2. However, higher TG level was not related with the incidence of systemic complication.

\section{Local complications}

The analysis of local complications, include acute peripancreatic fluid collection, pancreatic pseudocyst, acute necrotic collection, walled-off necrosis, and IPN, as shown in Table 5 . The results in Table 5 showed that, more IPN were found in the BAP patients (193 (45.4\%), $106(34.8 \%),(p=0.005))$, although similarity in acute necrotic collection (292 (68.7\%), 213 (69.8\%), $(p=$ 0.807)).

\section{Outcome}

The outcome comparison was shown in Table 6 . The two groups were not statistically different in terms of in-hospital mortality. Thanks to our four-step drainage strategy [6], the amount of patients who need surgery was less than $11 \%$.The length of hospital stay or ICU

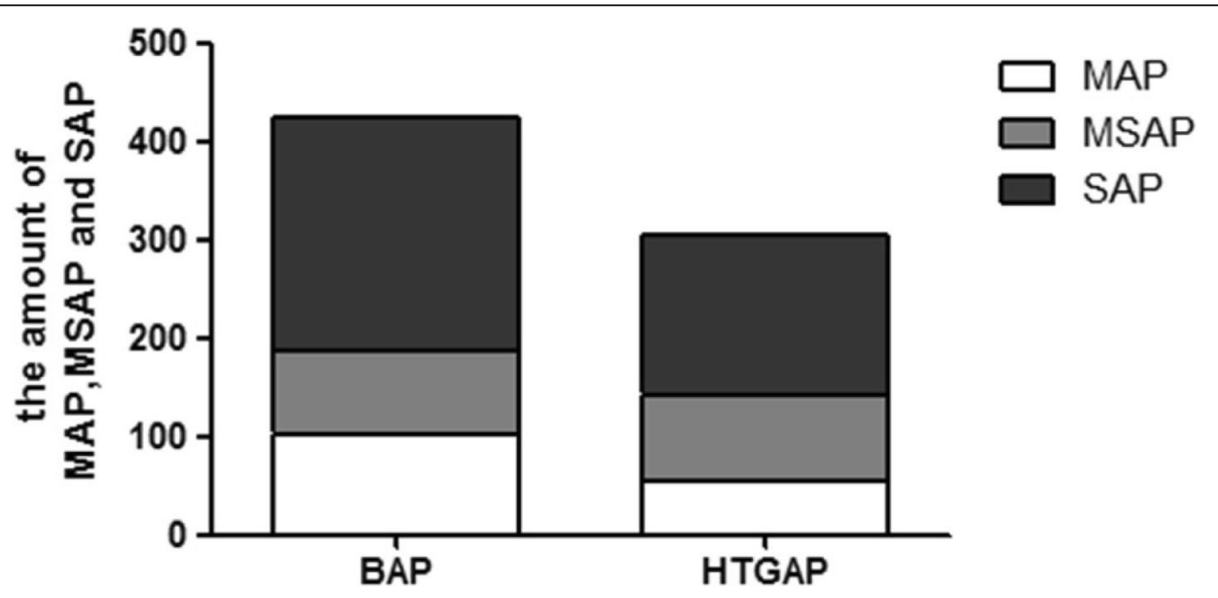

Fig. 2 The amount of MSAP, AP, SAP in BAP and HTG-AP group 
Table 2 Initial Laboratory data of the patients

\begin{tabular}{llll}
\hline Physiological Indexes & Biliary acute Pancreatitis $(n=425)$ & Hypertriglyceridemia acute Pancreatitis $(n=305)$ & $P$ value \\
\hline Amylase & $86(41,219)$ & $69(34,159.5)$ & 0.13 \\
Lipase & $295(107,706)$ & $355(127.5778 .5)$ & $10.4(7.7,13.6)$ \\
WBC & $11(7.5,14.9)$ & $152.1(87.6210 .3)$ & 0.599 \\
CRP & $114(47.4173 .1)$ & $98(51.7174 .1)$ & 0.306 \\
IL-6 & $60(30.4135 .9)$ & $1(0.2,3.4)$ & 0.01 \\
Procalcitonin(PCT) & $0.3(0.15,1.2)$ & $5.1(3.7,8.8)$ & 0.282 \\
Urine toxic nitrogen & $4.9(3.4,7.6)$ & $60(46,84)$ & 0.128 \\
Creatinine & $55(42,72)$ & $19.4(13.2,29.4)$ & 0.07 \\
Total bilirubin (TBil) & $19.4(13.1,31.1)$ & $13(4,28)$ & 0.031 \\
Conjugated bilirubin(DBil) & $13(4,36)$ & $25(19,39)$ & 0.381 \\
Alanine aminotransferase(ALT) & $37(23,73)$ & $31(20,50)$ & 0.365 \\
Aspartate transaminase(AST) & $30(21,52)$ & $106(85,133)$ & $P<0.01$ \\
Hemoglobin & $107(91.5127)$ & $178(119,244.5)$ & 0.408 \\
Blood platelet & $194(134,269)$ & $13.1(12.5,14.3)$ & 0.709 \\
Prothrombin time(PT) & $13.4(12.5,14.5)$ & $32(28,36.4)$ & 0.058 \\
Activated partial thromboplastin time(APT) & $34(29.9,39.7)$ & $3.6(2.0,7.1)$ & 0.58 \\
D-Dimer & $3.3(1.5,5.9)$ & & 0.147 \\
\hline WBC whit & & & 0.141 \\
\hline
\end{tabular}

WBC white blood cell, CRP C-reactive protein

stay between two group was similar and nearly $60 \%$ needed to be admitted to ICU.

\section{Discussion}

This retrospective study demonstrated significantly different clinical features between HTG-AP and BAP patients. The results indicated that HTG-AP patients were younger, more male, more ratio of high fat diet, higher
BMI and higher prevalence of AKI/ARDS/DVT/MODS, but, the BAP patients had higher incidence of IPN and chylous fistula. Moreover, the multivariate analysis showed that HTG-AP was independently associated with more AKI as adjusting by age, gender, body mass index, diabetic mellitus, fatty liver and high-fat diet, but not with ARDS and DVT. However, BMI was found to be independently associated with AKI, ARDS and DVT.

Table 3 Systemic complications between the patients

\begin{tabular}{llll}
\hline Variable & Biliary acute Pancreatitis $(n=425)$ & Hypertriglyceridemia acute Pancreatitis $(n=305)$ & $P$ value \\
\hline ARDS & $130(30.6 \%)$ & $116(38.0 \%)$ & 0.039 \\
AKI & $91(21.4 \%)$ & $105(34.4 \%)$ & $P<0.01$ \\
Intra-abdominal hypertension & $23(5.4 \%)$ & $28(9.2 \%)$ & 0.056 \\
Shock & $66(15.5 \%)$ & $49(16.1 \%)$ & 0.838 \\
Intra-abdominal hemorrhage & $56(13.2 \%)$ & $34(11.1 \%)$ & 0.427 \\
Sepsis & $39(9.2 \%)$ & $21(6.9 \%)$ & 0.278 \\
Portal vein thrombosis & $47(11.1 \%)$ & $26(8.5 \%)$ & 0.317 \\
Deep venous thrombosis & $27(6.4 \%)$ & $37(12.1 \%)$ & 0.008 \\
Acute hepatic injury & $45(10.6 \%)$ & $21(6.9 \%)$ & 0.090 \\
Gastrointestinal fistula & $75(17.6 \%)$ & $44(14.4 \%)$ & 0.265 \\
Digestive tract hemorrhage & $11(2.6 \%)$ & $8(2.6 \%)$ & 1 \\
Chylous fistula & $6(1.4 \%)$ & $0(0.0 \%)$ & 0.044 \\
Diarrhea & $9(2.1 \%)$ & $9(3.0 \%)$ & 0.479 \\
Ileus & $7(1.6 \%)$ & $11(3.6 \%)$ & 0.144 \\
MODS & $96(22.6 \%)$ & $91(29.8 \%)$ & 0.032 \\
\hline ARDS
\end{tabular}


Table 4 Multivariate analysis showing association of proposed risk factors with ARDS/AKI/DVT

\begin{tabular}{|c|c|c|c|c|c|c|}
\hline \multirow{2}{*}{$\begin{array}{l}\text { Multivariate } \\
\text { analysis }\end{array}$} & \multicolumn{2}{|l|}{ ARDS } & \multicolumn{2}{|l|}{ AKI } & \multicolumn{2}{|l|}{$\mathrm{DVT}$} \\
\hline & $\mathrm{OR}(95 \% \mathrm{Cl})$ & $P$ value & OR(95\% Cl) & $P$ value & $\mathrm{OR}(95 \% \mathrm{Cl})$ & $P$ value \\
\hline Etiology & $0.88(0.58,1,32)$ & 0.52 & $0.62(0.40,0.96)$ & 0.03 & $1.03(0.53,2.00)$ & 0.93 \\
\hline Age & $0.99(0.98,1.01)$ & 0.25 & $0.99(0.98,1.00)$ & 0.17 & $1.01(0.99,1.03)$ & 0.36 \\
\hline Gender & $0.93(0.67,1.29)$ & 0.66 & $0.69(0.48,0.99)$ & 0.04 & $1.45(0.84,2.50)$ & 0.19 \\
\hline BMI & $0.95(0.91,0.98)$ & 0.004 & $0.96(0.92,1.00)$ & 0.03 & $0.91(0.86,0.97)$ & 0.002 \\
\hline Diabetes mellitus & $1.38(0.92,2.07)$ & 0.12 & $1.34(0.87,2.05)$ & 0.18 & $0.85(0.46,1.57)$ & 0.60 \\
\hline Fatty liver & $0.99(0.68,1.45)$ & 0.98 & $0.79(0.54,1.17)$ & 0.24 & $0.67(0.38,1.19)$ & 0.17 \\
\hline High fat diet & $0.76(0.52,1.10)$ & 0.14 & $0.88(0.59,1.31)$ & 0.53 & $0.79(0.44,1.41)$ & 0.42 \\
\hline
\end{tabular}

ARDS acute respiratory distress syndrome, $A K I$ acute kidney injury, DVT deep venous thrombosis

The results suggested that higher incidence of AKI in HTG-AP was significantly and independently associated with the etiology, but more ARDS and DVT were more due to higher BMI.

HTG-AP is a rare but well-documented type of AP in Western countries $(1.3-5 \%)$ [7, 8]. Recently, accumulating data have shown that HTG-AP has become the second common cause of AP in China, with a reported incidence up to12.3\% in 2003 [9], 18.1\% in 2007 [10], and $25.6 \%$ in 2013 [11], much higher than that in Western countries. In our center, which is the largest AP referral center in China, HTG-AP accounted for about $30 \%$ from 2013 to 2016, being also the second leading cause of AP. Previous studies had compared HTG-AP with other common etiologies, but the results vary. Linares et al. [12] reported that patients with HTG-AP suffered a more severe clinical course than those with alcohol or gallstone-induced based on the index such as ICU admission, CRP, and Balthazar scores and similar findings were repeatedly reported in the literature [13]. Tai et al. [14] reported no significant difference in specific complications like ARDS, AKI, gastrointestinal bleeding and sepsis. Besides, clinical studies assessing the impact of the TG level on the severity of AP also showed conflicting results. Zhang et al. [15], S. Balachandra et al. [16] and Fortson MR et al. [7] showed no difference detected in severity, based on APACHE II scores or complications in patients with the level of TG. While some reports demonstrated that patients with high level of triglyceride may suffer worse clinical outcome. A previous study showed that the level of TG in
AP patients are independently and proportionally correlated with persistent organ failure regardless of etiology [17], in another study, AP patients with HTG (> $500 \mathrm{mg} /$ $\mathrm{dL}$ ) had higher $24 \mathrm{~h}$ APACHE II scores, more systemic complications and higher mortality [18].

The underlying pathogenesis of HTG-AP is not fully understood and the most widely accepted explanation hitherto is the "free fatty acids (FFA)" theory. It is assumed that at the onset of pancreatitis, a large amount of pancreatic lipase releases into the systemic circulation hydrolyzing serum TGs and adipose tissue, which would consequently generate high concentrations of free fatty acids with detergent properties. Free fatty acid micelle complexes injure the vascular endothelium and acinar cells of the pancreas, making internal environment increasingly acidic and ischemic, which triggers further free fatty acid toxicity and accelerate systemic inflammatory response [19]. Furthermore, direct tissue injury and lipotoxicity via mitochondrial stress [20-22] may cause the up-regulation of cytokines and the inflammatory cascade, predisposing to systemic inflammatory response [22].

In the complications of AP, the incidence of AKI was also found to be higher in HTG-AP in other reports [23]. Wu et al. indicated that TG may do a direct damage to renal parenchyma as reacting with pancreatic lipase around kidney tubules. The pancreatic enzymes assembled in glomerulus aggravates the damage of renal function [23]. Also Scheuer et al. reported that, the infiltrated TG in glomerular and tubulointerstitial could make the glomerulosclerosis grow worse in mouse model [24].

Table 5 Local complications between BAP and HTG-AP

\begin{tabular}{llll}
\hline Variable & Biliary acute Pancreatitis $(n=425)$ & Hypertriglyceridemia acute Pancreatitis $(n=305)$ & $P$ value \\
\hline Acute peripancreatic fluid collection & $120(28.2 \%)$ & $104(34.1 \%)$ & 0.104 \\
Pancreatic pseudocyst & $11(2.6 \%)$ & $8(2.6 \%)$ & 1 \\
Acute necrotic collection & $292(68.7 \%)$ & $213(69.8 \%)$ & 0.807 \\
Walled-off necrosis & $5(1.2 \%)$ & $3(1.0 \%)$ & 1 \\
Infected pancreatitis necrosis & $193(45.4 \%)$ & $106(34.8 \%)$ & 0.005 \\
\hline
\end{tabular}


Table 6 Outcome comparisons between the patients

\begin{tabular}{llll}
\hline Variable & Biliary acute Pancreatitis $(n=425)$ & Hypertriglyceridemia acute Pancreatitis $(n=305)$ & $P$ value \\
\hline Hospital mortality, no. & $36(8.5 \%)$ & $24(7.9 \%)$ & 0.787 \\
Need of surgery, no. & $39(9.2 \%)$ & $33(10.8 \%)$ & 0.529 \\
ICU admission & $260(61.2 \%)$ & $186(61.0 \%)$ & 1 \\
Length of ICU stay, days & $4(2,12)$ & $4(2,10.5)$ & 0.975 \\
Length of hospital stay, days & $9(4,22.5)$ & $9(5,23)$ & 0.58 \\
Cost, Thousand CHY & $48.7(22.8157 .8)$ & $51.0(27.9134 .6)$ & 0.623 \\
\hline
\end{tabular}

ICU intensive care unit

Moreover, the results in this study also showed that higher BMI was independently associated with AKI, ARDS and DVT in multivariate analysis. Up to date, quite a few articles had showed that BMI may increase the severity of AP from clinical findings and animal models [25]. A possible explanation is that adipose tissue appears as chronic inflammation releasing pro-inflammatory cytokines and obese individuals are more likely to have lifestyle-related chronic diseases/ respiratory problems $[25,26]$. Obesity has also been inferred associated with an increased risk of extrapancreatic complications such as shock, renal failure, respiratory insufficiency, and fatal outcome [27, 28].

Furthermore, the results found the BAP patients suffered more infected local complications than HTG-AP. In BAP patients, when the gallstones pass through the Vater ampulla, spasm, fibrosis and obstruction of the hepatopancreatic ampulla happens, resulting in biliopancreatic reflux and the exclusion of bile and pancreatic juices. Then the elevated levels of bile and pancreatic juices and activation of pancreatic enzymes are responsible for pancreatitis attacking ultimately in this abnormal physiological status [3]. The refluxing of infected bile transfer into the pancreatic duct may generate Escherichia coli and other bacteria leading to the infection [29].

\section{Conclusions}

In conclusion, this study showed significantly different clinical features between HTG-AP and BAP patients. HTG-AP patients suffered higher occurrence rates of AKI/ARDS/DVT/MODS, while IPN was more common in BAP patients. After the multivariate analysis adjusted by age, gender, BMI, diabetes, fatty liver and high fat diet, HTG-AP was found to be significantly and independently related with more incidence of AKI. However, higher BMI was found to be related with AKI, ARDS and DVT. Further, larger prospective studies should be performed to study the features between the different etiologies of AP and the effect of BMI on disease severity.

\section{Additional files}

Additional file 1: Figure S1. The standardized protocol for the fluid resuscitation treatment. (DOCX $213 \mathrm{~kb}$ )

Additional file 2: Table S1. Characteristics in three groups divided by triglyceride levels. (DOCX $15 \mathrm{~kb}$ )

Additional file 3: FigureS2. Proportion of systemic complication with three groups according to the value of TG level in patients with HTG-AP using the Cochran-Armitage trend test. A. Proportion of ARDS with three groups according to the value of TG level in patients with HTG-AP. B. Proportion of AKI with three groups according to the value of TG level in patients with HTG-AP. C. Proportion of DVT with three groups according to the value of TG level in patients with HTG-AP.

Cochran-Armitage test for trend was analyzed. (DOCX $26 \mathrm{~kb}$ )

\begin{abstract}
Abbreviations
AKl: Acute kidney injury; ALT: Alanine aminotransferase; AP: Acute pancreatitis; APACHE II: Acute physiology and chronic health evaluation II; ARDS: Acute respiratory distress syndrome; BAP: Biliary acute pancreatitis; BMI: Body mass Index; CECT: Contrast-enhanced computed tomography; CRP: C-reactive protein; DVT: Deep vein thrombosis; EUS: Endoscopic ultrasonography; FFA: Free fatty acids; HTG: Hypertriglyceridemia; HTGAP: Hypertriglyceridemia acute pancreatitis; ICU: Intensive Care Unit; IPN: Infected pancreatitis necrosis; IQR: Interquartile range; MAP: Mild acute pancreatitis; MODS: Multiple organ dysfunction syndrome; MRCP: Magnetic resonance cholangiopancreatography; MRI: Magnetic resonance imaging; MSAP: Moderate severe acute pancreatitis; SAP: Severe acute pancreatitis.; SICU: Surgical Intensive Care Unit
\end{abstract}

\section{Acknowledgements}

The authors are indebted to all doctors and researchers for the follow-up assessment and data collection during the study from the severe acute pancreatitis care center of Jinling Hospital, Medical School of Nanjing University.

\section{Funding}

The article processing charge was funded by National Natural Science Foundation of China (No.81570584). The article publication charge was funded by Social development project of Jiangsu Province(BE2015685), Medical Research Funding of PLA(AWS14C003). The funding bodies did not participate in the study design, data collection, data analysis, results interpretation or writing of the manuscript.

Availability of data and materials

Data for the analyses are available from the corresponding author on request.

\section{Authors' contributions}

XYL, LK: Study concept and design, JD, LM: Statistical analysis, BY, WJM: Acquisition of data, analysis and interpretation of data, XYL: Drafting of the manuscript, QY, WQL: Critical revision of the manuscript for important intellectual content, WQL and JSL: Study supervision. All authors have read and approved the final version of this manuscript, including the authorship. 


\section{Ethics approval and consent to participate}

The study was approved by the ethics committee of the Jinling Hospital, Medical School of Nanjing University. All participants consented to participate the study and the written informed consent was obtained from each subject.

\section{Consent for publication}

Not applicable.

\section{Competing interests}

The authors declare that they have no competing interests.

\section{Publisher's Note}

Springer Nature remains neutral with regard to jurisdictional claims in published maps and institutional affiliations.

Received: 16 August 2017 Accepted: 7 June 2018

Published online: 19 June 2018

\section{References}

1. Banks PA, Bollen TL, Dervenis C, Gooszen HG, Johnson CD, Sarr MG, Tsiotos GG, Vege SS, Acute pancreatitis classification working G. Classification of acute pancreatitis-2012: revision of the Atlanta classification and definitions by international consensus. Gut. 2013;62(1):102-11.

2. Dellinger EP, Forsmark CE, Layer P, Levy P, Maravi-Poma E, Petrov MS, Shimosegawa T, Siriwardena AK, Uomo G, Whitcomb DC, et al. Determinant-based classification of acute pancreatitis severity: an international multidisciplinary consultation. Ann Surg. 2012;256(6):875-80.

3. van Geenen EJM, van der Peet DL, Bhagirath P, Mulder CJJ, Bruno MJ. Etiology and diagnosis of acute biliary pancreatitis. Nat Rev Gastroenterol Hepatol. 2010;7(9):495-502.

4. Scherer J, Singh VP, Pitchumoni CS, Yadav D. Issues in hypertriglyceridemic pancreatitis: an update. J Clin Gastroenterol. 2014;48(3):195-203.

5. Tenner S, Baillie J, De Witt J, Vege SS, American College of G. American College of Gastroenterology guideline: management of acute pancreatitis. Am J Gastroenterol. 2013:108(9):1400-15. 1416

6. Tong Z, Ke L, Li B, Li G, Zhou J, Shen X, Li W, Li N, Li J. Negative pressure irrigation and endoscopic necrosectomy through man-made sinus tract in infected necrotizing pancreatitis: a technical report. BMC Surg. 2016;16:1.

7. Fortson MR, Freedman SN, Webster PD 3rd. Clinical assessment of hyperlipidemic pancreatitis. Am J Gastroenterol. 1995;90(12):2134-9.

8. Ivanova R, Puerta S, Garrido A, Cueto I, Ferro A, Ariza MJ, Cobos A, Gonzalez-Santos P, Valdivielso P. Triglyceride levels and apolipoprotein E polymorphism in patients with acute pancreatitis. Hepatobiliary Pancreat Dis Int. 2012;11(1):96-101.

9. Chang MC, Su CH, Sun MS, Huang SC, Chiu CT, Chen MC, Lee KT, Lin CC, Lin JT. Etiology of acute pancreatitis-a multi-center study in Taiwan. Hepatogastroenterology. 2003;50(53):1655-7.

10. Qian JM. Reviewing the etiology, diagnosis and treatment of acute pancreatitis in China. Zhonghua Nei Ke Za Zhi. 2007;46(12):979-80.

11. Yin G, Cang X, Yu G, Hu G, Ni J, Xiong J, Hu Y, Xing M, Chen C, Huang Y, et al. Different clinical presentations of Hyperlipidemic acute pancreatitis: a retrospective study. Pancreas. 2015;44(7):1105-10.

12. Lloret Linares C, Pelletier AL, Czernichow S, Vergnaud AC, BonnefontRousselot D, Levy P, Ruszniewski P, Bruckert E. Acute pancreatitis in a cohort of 129 patients referred for severe hypertriglyceridemia. Pancreas. 2008; 37(1):13-2.

13. Adiamah A, Psaltis E, Crook M, Lobo DN. A systematic review of the epidemiology, pathophysiology and current management of hyperlipidaemic pancreatitis. Clin Nutr. 2017.

14. Tai WP, Lin XC, Liu H, Wang CH, Wu J, Zhang NW, Chen W. A retrospective research of the characteristic of Hypertriglyceridemic pancreatitis in Beijing, China. Gastroenterol Res Pract. 2016;2016:6263095.

15. Zhang XL, Li F, Zhen YM, Li A, Fang Y. Clinical study of 224 patients with hypertriglyceridemia pancreatitis. Chin Med J. 2015;128(15):2045-9.

16. Balachandra S, Virlos IT, King NK, Siriwardana HP, France MW, Siriwardena AK. Hyperlipidaemia and outcome in acute pancreatitis. Int I Clin Pract. 2006;60(2):156-9.

17. Nawaz H, Koutroumpakis E, Easler J, Slivka A, Whitcomb DC, Singh VP, Yadav D, Papachristou GI. Elevated serum triglycerides are independently associated with persistent organ failure in acute pancreatitis. Am J Gastroenterol. 2015:110(10):1497-503.

18. Deng LH, Xue P, Xia Q, Yang XN, Wan MH. Effect of admission hypertriglyceridemia on the episodes of severe acute pancreatitis. World Gastroenterol. 2008;14(28):4558-61.

19. Samuel I, Zaheer S, Zaheer A. Bile-pancreatic juice exclusion increases p38MAPK activation and TNF-alpha production in ligation-induced acute pancreatitis in rats. Pancreatology. 2005;5(1):20-6.

20. Zeng Y, Wang X, Zhang W, Wu K, Ma J. Hypertriglyceridemia aggravates ER stress and pathogenesis of acute pancreatitis. Hepatogastroenterology. 2012:59(119):2318-26.

21. Chang YT, Chang MC, Su TC, Liang PC, Su YN, Kuo CH, Wei SC, Wong JM. Association of cystic fibrosis transmembrane conductance regulator (CFTR) mutation/variant/haplotype and tumor necrosis factor (TNF) promoter polymorphism in hyperlipidemic pancreatitis. Clin Chem. 2008:54(1):131-8.

22. Chang YT, Chang MC, Su TC, Liang PC, Su YN, Kuo CH, Wei SC, Wong JM. Lipoprotein lipase mutation S447X associated with pancreatic calcification and steatorrhea in hyperlipidemic pancreatitis. J Clin Gastroenterol. 2009; 43(6):591-6

23. Wu C, Ke L, Tong Z, Li B, Zou L, Li W, Li N, Li J. Hypertriglyceridemia is a risk factor for acute kidney injury in the early phase of acute pancreatitis. Pancreas. 2014:43(8):1312-6.

24. Scheuer H, Gwinner W, Hohbach J, Grone EF, Brandes RP, Malle E, Olbricht CJ, Walli AK, Grone HJ. Oxidant stress in hyperlipidemia-induced renal damage. Am J Physiol Renal Physiol. 2000;278(1):F63-74.

25. Ikeura T, Kato K, Takaoka M, Shimatani M, Kishimoto M, Nishi K, Kariya S, Okazaki K. A body mass index $>/=25 \mathrm{~kg} / \mathrm{m}(2)$ is associated with a poor prognosis in patients with acute pancreatitis: a study of Japanese patients. Hepatobiliary Pancreat Dis Int. 2017;16(6):645-51.

26. Hersoug LG, Moller P, Loft S. Gut microbiota-derived lipopolysaccharide uptake and trafficking to adipose tissue: implications for inflammation and obesity. Obes Rev. 2016;17(4):297-312.

27. Lankisch PG, Schirren CA. Increased body weight as a prognostic parameter for complications in the course of acute pancreatitis. Pancreas. 1990;5(5): 626-9.

28. Blomgren KB, Sundstrom A, Steineck G, Wiholm BE. Obesity and treatment of diabetes with glyburide may both be risk factors for acute pancreatitis. Diabetes Care. 2002;25(2):298-302.

29. Arendt R, Liebe S, Erdmann K. Biliary pancreatitis-pathogenesis, therapy, results. Z Gesamte Inn Med. 1989:44(13):401-4.

\section{Ready to submit your research? Choose BMC and benefit from:}

- fast, convenient online submission

- thorough peer review by experienced researchers in your field

- rapid publication on acceptance

- support for research data, including large and complex data types

- gold Open Access which fosters wider collaboration and increased citations

- maximum visibility for your research: over $100 \mathrm{M}$ website views per year

At BMC, research is always in progress.

Learn more biomedcentral.com/submissions 